\title{
Optimal Claim Settlement Strategies under Constraint of Cap on Claim Loss
}

\author{
Hong Mao ${ }^{1}$ (D) and Krzysztof Ostaszewski ${ }^{2, *}$ (D) \\ 1 School of Economics and Management, Shanghai Second Polytechnic University, Shanghai 201209, China; \\ hmaoi@vip.126.com \\ 2 Department of of Mathematics, Illinois State University, Normal, IL 61790-4520, USA \\ * Correspondence: krzysio@ilstu.edu; Tel.: +1-309-287-1711
}

check for updates

Citation: Mao, H.; Ostaszewski, K. Optimal Claim Settlement Strategies under Constraint of Cap on Claim Loss. Mathematics 2021, 9, 3284. https://doi.org/10.3390/ math9243284

Academic Editors: Eric Ulm, Budhi Surya and Bahram Adrangi

Received: 25 October 2021

Accepted: 14 December 2021

Published: 17 December 2021

Publisher's Note: MDPI stays neutral with regard to jurisdictional claims in published maps and institutional affiliations.

Copyright: (C) 2021 by the authors. Licensee MDPI, Basel, Switzerland. This article is an open access article distributed under the terms and conditions of the Creative Commons Attribution (CC BY) license (https:// creativecommons.org/licenses/by/ $4.0 /)$.

\begin{abstract}
In this paper, we examine the question of how to devise an optimal insurance claim settlement scheme under the constraint of a cap on the amount of the claim payment. We establish objective functions to maximize the net benefit due to exaggerated claims while at the same time maximizing the total expected wealth of the insured. Then, we establish a dual objective function to minimize the total expected loss, including the perspective of the insurer. Finally, we illustrate applications of our work and provide numerical analysis of it along with an example.
\end{abstract}

Keywords: insurance contract; insurance claim; claim cap; insured loss

\section{Introduction}

Deductibles and policy upper limits are standard methods of controlling moral hazard in insurance contracts (see for example Borch [1]). The subject is widely studied in the existing insurance literature and is of utmost practical significance. Cummins and Mahul [2] examine the demand for insurance when the indemnity schedule is subject to an upper limit. The optimal contract is shown to be full insurance above a deductible, up to a cap. Mossin's Theorem generally states that any risk-averse individual would prefer actuarially fair insurance to no insurance, and Schlesinger [3] showed that it is also valid for the class of upper limit insurance contracts (this specific version of the theorem was not proven before although, in general, Mossin's Theorem is well known for the class of coinsurance contracts and for contracts with deductibles). Huang and Tzeng [4] discuss how a policymaker can choose a tax deduction rate to maximize the weighted average of the consumers' expected utility and the insurer's expected value. Mao et al. [5] studied a risk-based valuation model of deposit insurance that accounted for the investment policy of deposit insurers, deductibles, and regulatory capital requirement in the pricing of deposit insurance. Their work also presented an upper limit for claim payments that is a declining exponential function (i.e., an exponential function with a negative exponent) of a bank's insolvency probability, in order to mitigate the problem of moral hazard inherent in deposit insurance.

Regarding the optimization of claim settlement strategy for liability insurance, the pioneering work was that of Crocker and Morgan [6], who applied an incentive contracting approach to characterize optimal contracts when insured individuals possess private information about their losses and are able to misrepresent permanently their loss magnitudes by engaging in falsification of claims. They showed that while efficient agreements necessarily induce some falsification, the extent of claims inflation is partially mitigated by an indemnification schedule that over-compensates small losses while underpaying larger ones. In their later work, Crocker and Tennyson [7] examined the optimal claims settlement strategy for a liability insurer when claimants can permanently misrepresent their losses by engaging in costly claims falsification and exaggerating their claim amount. They suggest that liability insurers optimally choose claims payment strategies to lessen a claimant's incentive to exaggerate losses. 
Caps on damage awards are among the key ideas of proposed reform of the tort system, especially in the United States. There is extensive legal research literature on the issue of imposing caps on liability; see for example Bambauer and Roth [8], Klick and MacDonald [9], and many others, as well as Born [10] for a combined legal and insurance perspective. However, the perspective of our paper is not legal, but rather the mathematical modeling of often legally imposed caps on liability payments.

In addition to caps imposed legally by legislation seeking to limit tort awards, caps also arise naturally in insurance and reinsurance via the use of policy limits and maximum payments (see for example Mert and Selcuk-Kestel's [11] work on such caps in the context of stochastic models of claims processes). Mao et al. [12-15] also consider models with contract modifications including caps, although not in the context of caps imposed by legislation or regulation. Literature on mathematical models in the context of caps imposed by legislation or regulation is limited, as most researchers turn their attention to the legal issues involved in the context of tort reform proposals.

Mao et al. [10] established models to capture both claimant and the insurer behavior under a system where damages are capped in a manner resembling caps imposed by law. They determined the optimal level of such caps by maximizing the sum of claimant and insurer benefits.

However, three shortcomings exist in the models. One is that the quadratic cost functions of the deviation of the real loss away from $\operatorname{cap} \theta$ is not an ideal function to describe the direct relationship between an exaggerated amount of damage claim and the real one, and it is difficult to accurately estimate the cost parameters as the cost function is not directly related to the exaggerated amount of the claim. Another shortcoming is that simply using the quadratic cost functions of the deviation of the real loss from cap $\theta$ may not produce the optimal solution. A third is that the optimization model of the paper did not consider indirect costs resulting from setting an upper limit.

In this current work, we have two main contributions to the literature on the subject. First, in order to address the aforementioned defects, we assume new cost functions. The new cost functions consider the impact of an exaggerated claim filed by the claimant, the cost of claim falsification on the indemnification amount, and other indirect costs occurring to both insurers and claimants. Second, we use dual objective functions to maximize the net benefit to the claimant from exaggerating the claim amount (minus any indirect loss to the claimant due to the cap) while minimizing the expected loss of the insurer, in order to lessen the claimant's incentive to exaggerate losses and reduce the cost to the insurer both due to falsification and indirectly due to setting the upper limit.

In this paper, we first establish two simultaneous objective functions for the insured: (i) maximizing the claimant's net benefit resulting from exaggerating the claim amount, and (ii) maximizing the expected total wealth of the insured; this refers to the benefit obtained by the insured after any costs incurred, as defined below in Equation (1). Subsequently, we establish a dual objective function of the insurer (compare with Owen [16]), (i) minimizing the expected loss of the insurer, so as to lessen a claimant's incentive to exaggerate losses, and (ii) reducing the costs of falsification and of other indirect costs related to setting up the cap. By solving two objective functions for the insured and the dual objective function for the insurer, we can find the optimal indemnification schedule vs. the optimal cap. In contrast with Cracker and Tennyson [7], in our dual objectives analysis we consider the effect of a cap on the indemnification amount, and we use the empirical results from Mao et al. [12] by fitting the probability function using empirical data from the United States. We also use a function of the indemnification amount which is different from the one used by Cracker and Morgan [6] in order to ensure that the indemnification amount is always greater than or equal to the real loss when a cap is set. The optimization scheme aims both to determine the optimal indemnification amount when the real damage is smaller than the cap, and to determine the optimal cap in order to avoid excessive claim compensation. 
After this Introduction, the paper is organized as follows: the model and optimization methodology are introduced and set up in Section 2; Section 3 discusses the numerical analysis; and Section 4 concludes the article.

\section{Model Based on Incentive and Compensative Mechanism}

We begin by presenting the notation used:

$X$ : Real loss of the claimant;

$s$ : The claim filed by the claimant;

$C_{1}$ : The direct and indirect costs that the claimant incurs in the process of submitting the claim;

$a, b$ : Constants used as parameters for models of costs of the insured and the insurer;

$\xi_{1}$ : Cost parameter of the insured;

$C_{2}$ : The direct and indirect costs that the insurer incurs in the process of processing the claim;

$\xi_{2}$ : Cost parameter of the insurer;

$\alpha$ and $\beta$ : decision variables used to determine strategies of the insured and the insurer;

$E\left(Y_{1}\right)$ : the expected wealth of the claimant;

$E(Z)$ : the expected loss of the insurer;

$f(x)$ : the probability density function;

$\theta$ : the cap on claims payments, for example, a cap on liability payments imposed by law.

In a manner similar to the approach of Cracker and Tennyson [7], we consider an environment in which insurers face claimants who may expend resources to exaggerate their claim losses; in response to this challenge insurers seek an optimal settlement strategy that lessens claim falsification by systematically underpaying those categories of claims where the cost to claimants of falsification is the lowest. We consider third-party liability insurance, and we assume the claimant and insurer to be risk-neutral. Third-party liability insurance is, in practice, the type of insurance where claim exaggeration is the greatest concern. In third-party liability insurance, the insurance company pays the claims on behalf of the insured, but the payment is made to a third party which is deemed (in a court case or in some form of arbitration or negotiation) to be owed payment of damages by the insured. An example of such a situation would be a case where a firm (the insured) creates a product or delivers a service that is determined to have done harm to the user of that product or service (the third party) and the firm is deemed to be liable for that harm; the firm has purchased third-party liability insurance, and hence the payment for that liability will be made by the insurance company.

The claimant has a real loss of $X$ (a random variable), which is fully known only to the claimant. However, the claimant files a claim of $s$ (with $s \geq X$ ). Assume that the indemnification payment made by the insurer to the insured, denoted by $I(s(x), x)$, is an increasing function of $s$ (with $s \geq X$ ), and must be less than or equal to $s$. If the insurer can identify the real amount of damage, then $I(s(X), X)=X$. When the indemnification amount is greater than the real amount of loss, $I(s(X), X)-X$ is the exaggerated claim amount realized for claimant. Our optimization process is built on establishing three objective functions:

(1) maximizing the net benefit to the insured due to exaggeration of the claim;

(2) maximizing the expected total wealth of the claimant;

(3) maximizing the expected total wealth of the insurer.

Next, we discuss the optimization models from the perspective of the insured.

Let the net benefit to the insured due to engaging in claim inflation be

$$
y_{1}=I(s(X), X)-C_{1}\left(\xi_{1}, s(X), X\right)
$$

where $C_{1}\left(\xi_{1}, s(X), X\right)$ represents the costs that the claimant incurs for the direct and indirect expense of setting up a claim. We model these costs as a function of $s, X$, and $\xi_{1}$, with $\xi_{1} \geq 0$, where $\xi_{1}$ is the cost parameter of the claimant. We assume that the claim costs increase strictly with the deviation of $s(X)$ away from real loss and the indirect cost of setting up 
the cap, as a declining exponential function (i.e., an exponential function with a negative exponent) of the upper limit denoted by $\theta$. Note that Abolmohammadi et al. [17] extended the cost function based on Taguchi's social loss function to linear, quadratic, exponential, and Linex. Here, the new cost functions reflect the fact that a rational insured would not exaggerate the claim amount when the loss exceeds the cap, and that there is still a cost to set up the cap if this happens; for example, an intangible loss due to delayed medical treatment in the case of bodily injury to the insured happens due to less indemnification and lower insurance protection due to reduced insurance demand. To avoid the trivial case, we require that $\theta>0$. It is also important to note that although setting a cap on claim losses will reduce claim payment by the insurer, it will increase claimants' willingness to exaggerate a claim and at the same time increase indirect costs to claimants. We represent these costs with the following specific function:

$$
C_{1}\left(\xi_{1}, s(X), X, \theta\right)=\left\{\begin{array}{cl}
\xi_{1}(s(X)-X)^{2} & \text { when } X \leq s(X) \\
\xi_{1} e^{-a \theta+b} & \text { otherwise }
\end{array}\right.
$$

where $a$ and $b$ are constants used to determine the cost of the insured and insurer. In order to ensure that $I(s(X))>X$, we appropriately amend the definition of $I(s(X))$ based on the approach of Crocker and Tennyson [7] as

$$
I(s)= \begin{cases}\frac{s-(1-\beta) \alpha}{\beta} & \text { when } s>\frac{s-(1-\beta) \alpha}{\beta}(\text { or } s<\alpha) \\ s & \text { otherwise, }\end{cases}
$$

where $0<\beta \leq 1$ and $\alpha>0$; here $\alpha, \beta$ are decision variables/parameters, which are used to establish function $I(s(X), X)$ and determine the strategy of the insurer and the insured.

Note that the second case of (3) is meaningless, as when $I(s(X), X)=s(X)$, it follows that $C_{2}\left(\xi_{1}, s(X), X\right)=0$, see Equation (26) further in this paper.

We will now write $x$ for specific values of the random variable $X$, and work on optimization design and structure. Consider the first objective function, maximizing the net benefit to the insured resulting from engaging in claim inflation:

$$
\operatorname{Max} y_{1}=I(s(x), x)-C_{1}\left(\xi_{1}, s(x), x\right)
$$

Then, the first order condition (i.e., condition based on the first derivative) is

$$
\frac{\partial I(s(x), x)}{\partial s}-\frac{\partial C_{1}\left(\xi_{1}, s(x), x, \theta\right)}{\partial s}=0
$$

Substituting Equation (2) into Equation (5), we obtain

$$
\frac{\partial y_{1}}{\partial s}=\frac{\partial I(s(x), x)}{\partial s}-2 \xi_{1}(s-x)=0
$$

Equation (6) indicates that the claimant will engage in claim inflation as long as the marginal return on increased claiming $\frac{\partial I(s)}{\partial s}$ is positive. By taking the partial derivative of (3) with respect to $s$, we also obtain

$$
\frac{\partial I(s(x), x)}{\partial s}=\frac{1}{\beta}
$$

By combining (3), (6) and (7), we redefine $s$ and $I$ as

$$
s(x, \beta)=\frac{1}{2 \xi_{1} \beta}+x,
$$


and

$$
I(s(x, \beta), \alpha)=\frac{\frac{1}{2 \xi_{1} \beta}+x-(1-\beta) \alpha}{\beta} .
$$

Note the use of the parameters $\alpha$ and $\beta$ in this formulation. While these parameters may not be immediately intuitive, they provide a well-functioning model and behave properly at the boundaries, for example when $\beta=1, I(s(x, \beta), \alpha)=s(x, \beta)$.

Equations (8) and (9) indicate that the claim filed by a claimant $s$ and the indemnity amount paid by an insured I will depend on not just the amount of real loss $x$, but also on the cost parameter of falsification, $\xi_{1}$.

Considering the second objective of the claimant, which is to maximize their total expected wealth, let $E\left(Y_{1}(\alpha, \beta, \theta)\right)$ be the expected terminal wealth of the claimant. Using that notation, we can write:

$$
\begin{aligned}
E\left(Y_{1}(\alpha, \beta, \theta)\right) & =\int_{0}^{\theta}\left(W_{Y_{1}}-C_{1}\left(\xi_{1}, s(x, \beta), x\right)+I(s(x, \beta))\right) f(x) d x+ \\
& +\int_{0}^{\theta}\left(W_{Y_{1}}-C_{1}\left(\xi_{1}, \theta\right)+\theta-x\right) f(x) d x
\end{aligned}
$$

where $W_{Y_{1}}$ is the claimant's initial wealth.

Equation (10) corresponds to two different situations:

(i) The point $\hat{x}$ defined by the condition $I(x=\hat{x})=s(x=\hat{x})$, is less than or equal to $\theta$ (recall that $\theta$ is the cap on claims payments, for example, a cap on liability payments imposed by law), i.e., $\hat{x}=\alpha-\frac{1}{2 \xi_{1} \beta} \leq \theta$;

(ii) $\hat{x}=\alpha-\frac{1}{2 \xi_{1} \beta}>\theta$.

For the second case, denoted by (ii), the first order conditions of the Lagrange equation are

$$
\begin{gathered}
\frac{\partial E\left(Y_{1}(\alpha, \beta, \theta)\right)}{\partial \theta}=\frac{\partial}{\partial \theta}\left(\begin{array}{l}
\int_{0}^{\hat{x}}\left((I-x)-\xi_{1}(s-x)^{2}\right) f(x) d x \\
+\int_{\hat{x}}^{\theta}(s-x) f(x) d x+\int_{\theta}^{+\infty}\left((\theta-x)+e^{-a \theta+b}\right) f(x) d x
\end{array}\right) \\
=(s(\theta)-\theta) f(\theta)+\int_{\theta}^{+\infty}\left(1-a e^{-a \theta+b}\right) f(x) d x-e^{-a \theta+b} f(\theta)+\lambda_{1}=0
\end{gathered}
$$

and

$$
\frac{\partial E\left(Y_{1}(\alpha, \beta, \theta)\right)}{\partial \lambda_{1}}=\theta-\alpha+\frac{1}{2 \xi_{1} \beta}=0 .
$$

It is clear that Equation (12) cannot be satisfied when

$$
\theta>\alpha-\frac{1}{2 \xi_{1} \beta} .
$$

In the second situation (ii), the indemnification of the insured $I(s(x))$ is either less than the real loss (damage) or greater than the exaggerated claim amount filed by the insured. These two claim schemes are not feasible, and we will not discuss them further as they produce no optimal solutions. Therefore, we only consider the first situation. Maximization of $E\left(Y_{1}\right)$ is equivalent to maximizing

$$
\begin{aligned}
& E\left(Y_{1}(\alpha, \beta, \theta)\right)=\int_{0}^{\theta}\left(I-x-\xi_{1}(s-x)^{2}\right) f(x) d x+\int_{\theta}^{+\infty}\left((\theta-x)-\xi_{1} e^{-a \theta+b}\right) f(x) d x \\
& \text { subject to } \theta \leq \alpha-\frac{1}{2 \xi_{1} \beta} .
\end{aligned}
$$


By using Leibniz' rule, we get first and second order conditions of the Lagrange equation as follows:

$$
\begin{aligned}
\frac{\partial L\left(E\left(Y_{1}\right)\right)}{\partial \theta} & =\left(I(\theta)-\xi_{1}(s(\theta)-\theta)^{2}\right) f(\theta)+ \\
& +\int_{\theta}^{+\infty}\left(1+a \xi_{1} e^{-a \theta+b}\right) f(x) d x-\left(\theta-\xi_{1} e^{-a \theta+b}\right) f(\theta)+\lambda_{1}=0,
\end{aligned}
$$

where

$$
I(\theta)=\frac{\theta+\frac{1}{2 \xi_{1} \beta}-\alpha(1-\beta)}{\beta} \text { and } s(\theta)=\theta+\frac{1}{2 \xi_{1} \beta} .
$$

Furthermore,

$$
\begin{gathered}
\frac{\partial L\left(E\left(Y_{1}\right)\right)}{\partial \beta}=\int_{0}^{\theta}\left(\frac{\partial I(s)}{\partial \beta}-2 \xi_{1}(s-x) \frac{\partial s}{\partial \beta}\right) f(x) d x \int_{\theta}^{+\infty} f(x) d x-\lambda_{1} \frac{1}{2 \xi_{1} \beta^{2}}=0 \\
\frac{\partial L\left(E\left(Y_{1}\right)\right.}{\partial \lambda}=\theta-\alpha+\frac{1}{2 \xi_{1} \beta}=0 .
\end{gathered}
$$

In addition,

$$
\begin{aligned}
\frac{\partial^{2} E\left(Y_{1}\right)}{\partial \theta^{2}} & =\left(\frac{\partial I(\theta)}{\partial \theta}-2\left(1+a \xi_{1} e^{-a \theta+b}\right)\right) f(\theta) \\
& +\left(I(\theta)-\xi_{1}(s(\theta)-\theta)^{2}-\left(\theta-\xi_{1} e^{-a \theta+b}\right)\right) \frac{\partial f(\theta)}{\partial \theta} \\
& -\int_{\theta}^{+\infty} a^{2} \xi_{1} e^{-a \theta+b} f(x) d x>0
\end{aligned}
$$

and

$$
\begin{aligned}
& \frac{\partial^{2} L\left(E\left(Y_{1}\right)\right)}{\partial \beta^{2}}=\int_{0}^{\theta} \frac{\partial I^{2}(s)}{\partial \beta^{2}} f(x) d x-\int_{0}^{\theta} 2 \xi_{1}\left(\begin{array}{c}
(s-I)\left(\frac{\partial^{2} s}{\partial \beta^{2}}-\frac{\partial^{2} I}{\partial \beta^{2}}\right) \\
+\left(\frac{\partial s}{\partial \beta}-\frac{\partial I}{\partial \beta}\right)^{2}
\end{array}\right) f(x) d x+\frac{\lambda_{1}}{\xi_{1} \beta^{3}}>0, \\
& \text { where } \begin{array}{c}
\frac{\partial I(s)}{\partial \beta}=\frac{\partial s}{\partial \beta} \frac{1}{\beta}+\frac{\alpha-s}{\beta^{2}}, \frac{\partial s}{\partial \beta}=-\frac{1}{2 \xi_{1} \beta^{2}}, \\
\frac{\partial^{2} I(s)}{\partial \beta^{2}}=\frac{\partial^{2} s}{\partial \beta^{2}} \frac{1}{\beta}-\frac{2(\alpha-s)}{\beta^{3}}-\frac{2}{\beta^{2}} \frac{\partial s}{\partial \beta}, \frac{\partial^{2} s}{\partial \beta^{2}}=\frac{1}{2 \xi_{1} \beta^{3}},
\end{array}
\end{aligned}
$$

and

$$
\theta=\alpha-\frac{1}{2 \xi_{1} \beta} .
$$

By combining Equations (15)-(18) we obtain

$$
\begin{gathered}
\left(\alpha-\xi_{1}\left(\frac{1}{2 \xi_{1} \beta}\right)^{2}-\left(\theta-\xi_{1} e^{-a \theta+b}\right)\right) f(\theta)+\int_{\theta}^{+\infty}\left(1+a \xi_{1} e^{-a \theta+b}\right) f(x) d x \\
+2 \xi_{1} \beta^{2}\left(\int_{\theta}^{+\infty} f(x) d x+\frac{1}{\beta^{2}}\left(\int_{0}^{\theta}\left(\alpha-x-\frac{1}{2 \xi_{1} \beta}\right) f(x) d x\right)\right)=0
\end{gathered}
$$

where $\theta=\alpha-\frac{1}{2 \tilde{\xi}_{1} \beta}$.

Let $E(Z(\alpha, \beta, \theta))$ be the expected loss of the insurer, including the expected indemnification amount and the cost of litigation and settlement. Assume that liability insurers optimally choose claims payment and upper limit strategies to lessen a claimant's incentive to exaggerate losses, which depends on the trade-off between the insurers' cost of litigation and settlement in order to decrease the inflated claim filed by the claimant, the final claim payment settled, and all indirect cost resulting from setting up the upper limit of the claim loss. 
Consider the dual objective function expressed in Equation (4), i.e., minimization of the expected loss of the insurer:

Min

$$
\begin{aligned}
E(Z(\alpha, \beta, \theta)) & =\int_{0}^{\theta}\left(C_{2}\left(\xi_{2}, s(x, \beta), I(s(x, \beta), \alpha, x)+I(s(x, \beta), \alpha, \beta)-x\right) f(x) d x\right. \\
& +\int_{\theta}^{+\infty}\left(C_{2}\left(\xi_{2}, \theta\right)+\theta-x\right) f(x) d x
\end{aligned}
$$

Subject to : $\theta \leq \alpha-\frac{1}{2 \xi_{1} \beta}$.

While this may seem like a simplification of the reality of costs (all economic costs, not necessarily just accounting costs) incurred by the insurer, we believe this is a reasonably good model of economic reality. $C_{2}\left(\xi_{2}, I(s(x, \beta), x), s(x, \beta), \theta\right)$ is the insurer's cost of investigation of possible falsification of the claim by the claimant and other indirect loss due to setting the cap, such as the loss resulting from the reduced number of customers and increased marketing cost in order to make up the deficiency in demand. We set it up as a declining exponential function (i.e., an exponential function with a negative exponent) of the cap, $\theta$. In addition, we consider it to be a function of $x$ and $\xi_{2}, a$ and $b$ with $\xi_{2}>0, a>0$ and $b>0$, with $\xi_{2}, a$, and $b$ being the cost parameters to be chosen at the model user's discretion. Then, the insurer's cost function under a regime of a cap on claims and/or damages payouts is expressed as

$$
C_{2}\left(\xi_{2}, I(s(x, \beta), x), s(x, \beta), \theta\right)=\left\{\begin{array}{cl}
\xi_{2}(s-I)^{2} & \text { when } x<\theta \text { and } s>I \\
0 & \text { when } x=\theta \text { and } s=I \\
\xi_{2} e^{-a \theta+b} & \text { otherwise. }
\end{array}\right.
$$

By combining Equation (15) with objective function (14), the dual objective function can be written as

$\operatorname{Min} E(Z(\alpha, \beta, \theta))=\int_{0}^{\theta}\left(I(s(x, \beta), \alpha, \beta)-x+\xi_{2}(s-I)^{2}\right) f(x) d x+\int_{\theta}^{+\infty}\left(\theta-x+\xi_{2} e^{-a \theta+b}\right) f(x) d x$

By using Leibniz' rule, we obtain the first and second order conditions of the Lagrange equation as follows:

$$
\begin{aligned}
& \frac{\partial L(E(Z))}{\partial \theta}=I(\theta) f(\theta)+\int_{\theta}^{+\infty}\left(1-a \xi_{2} e^{-a \theta+b}\right) f(x) d x-\left(\theta+\xi_{2} e^{-a \theta+b}\right) f(\theta)+\lambda_{2}=0 \\
& \frac{\partial L(E(Z))}{\partial \alpha}=\int_{0}^{\theta} \frac{\partial I(s)}{\partial \alpha} f(x) d x+\int_{0}^{\theta} 2 \xi_{2} \frac{(1-\beta)}{\beta}(\alpha-s)\left(1-\frac{\partial s}{\partial \alpha}\right) f(x) d x+\int_{\theta}^{+\infty} f(x) d x-\lambda_{2}=0 \\
& \text { and } \\
& \qquad \frac{\partial L(E(Z))}{\partial \lambda_{2}}=\theta-\alpha+\frac{1}{2 \xi_{1} \beta}=0, \\
& \text { where } \begin{array}{c}
\frac{\partial I(s)}{\partial \alpha}=\frac{1}{\beta}\left(\frac{\partial s}{\partial \alpha}-(1-\beta)\right), \frac{\partial s}{\partial \alpha}=0 \text { and } \theta=\alpha-\frac{1}{2 \xi_{1} \beta} . \\
\frac{\partial L^{2}(E(Z))}{\partial \theta^{2}}=\left(\frac{\partial I(\theta)}{\partial \theta}-2\left(1-a \xi_{2} e^{-a \theta+b}\right)\right) f(\theta)+\left(I(\theta)-\theta-\xi_{2} e^{-a \theta+b}\right) \frac{\partial f(\theta)}{\partial \theta} \\
+\int_{\theta}^{+\infty} a^{2} \xi_{1} e^{-a \theta+b} f(x) d x>0
\end{array}
\end{aligned}
$$


where

$$
s(\theta)=\theta+\frac{1}{2 \xi_{1} \beta}, I(\theta)=\frac{s(\theta)-\alpha(1-\beta)}{\beta}, \frac{\partial s(\theta)}{\partial \theta}=1 \text {, and } \frac{\partial I(\theta)}{\partial \theta}=\frac{1}{\beta} .
$$

Additionally,

$$
\begin{gathered}
\frac{\partial^{2}(Z)}{\partial \alpha^{2}}=\int_{0}^{\theta} \frac{\partial^{2} I(s)}{\partial \alpha^{2}} f(x) d x+\int_{0}^{\theta} 2 \xi_{2} \frac{(1-\beta)}{\beta}\left((\alpha-s)\left(-\frac{\partial^{2} s}{\partial^{2} \alpha}\right)+\left(1-\frac{\partial s}{\partial \alpha}\right)^{2}\right) f(x) d x>0 \\
\text { with } \\
\frac{\partial^{2} I(s)}{\partial \alpha^{2}}=\frac{\partial^{2} s}{\partial \alpha^{2}} \frac{1}{\beta} \text { and } \frac{\partial s}{\partial \alpha}=\frac{\partial^{2} s}{\partial \alpha^{2}}=0 .
\end{gathered}
$$

We see that clearly that the condition $\frac{\partial^{2}(Z)}{\partial \alpha^{2}}>0$ holds.

By combining Equations (26), (27), (25) and (28), we obtain

$$
\begin{aligned}
\alpha f(\theta) & +\int_{\theta}^{+\infty}\left(1-a \xi_{2} e^{-a \theta+b}\right) f(x) d x-\left(\theta+\xi_{2} e^{-a \theta+b}\right) f(\theta)+\int_{\theta}^{+\infty} f(x) d x \\
& +\int_{0}^{\theta}\left(1-\frac{1}{\beta}\right)\left(1+2 \xi_{2}\left(\alpha-x-\frac{1}{2 \xi_{1} \beta}\right)\right) f(x) d x=0,
\end{aligned}
$$

where $\theta=\alpha-\frac{1}{2 \xi_{1} \beta}$.

By solving the system of Equations (22), (30) and (36) using the numerical method, we can obtain optimal solutions for $\left(\alpha^{*}, \beta^{*}, \theta^{*}\right)$. Moreover, based on Equation (9), we can obtain the expected value of the indemnification amount, expressed as

$$
E(I(s, x))=\int_{0}^{\theta} \frac{x+\frac{1}{2 \xi_{1} \beta}-(1-\beta) \alpha}{\beta} f(x) d x+\int_{\theta}^{+\infty} \theta f(x) d x .
$$

Entering $\left(\theta^{*}, \alpha^{*}, \beta^{*}\right)$ into Equation (37), we obtain the optimal cap and optimal expected value of indemnification amount $E\left(I^{*}\left(\theta^{*}, \alpha^{*}, \beta^{*}\right)\right)$.

The above equations illustrate that the optimal solutions for $\left(\theta^{*}, \alpha^{*}, \beta^{*}\right)$ and the optimal expected value of indemnification amount $E\left(I^{*}\right)$ are dependent on the cost parameters of falsification and setting up the cap on claim losses for both the insured and the insurer, $\xi_{1}, \xi_{2}, k_{1}$, and $k_{2}$, and on the probability distribution of real damage.

\section{Results}

\section{Numerical Analysis}

In a manner similar to Example 1 in Mao et al. [12], we consider a lognormal loss distribution with parameters $\mu=9.294$ and $\sigma=1.627$. In this case, (note that ln denotes the natural logarithm) the loss density is

$$
f(x)=\frac{1}{x \sigma \sqrt{2 \pi}} e^{-\frac{(\ln x-\mu)^{2}}{2 \sigma^{2}}}, x>0 .
$$


Using the density given in Equation (38) in Equations (22) and (36), and substituting a variable $t=\frac{\ln x-\mu}{\sigma}$ in Equations (22) and (36), we obtain the following first-order conditions:

$$
\begin{gathered}
\left(\left(\alpha-\xi_{1}\left(\frac{1}{2 \xi_{1} \beta}\right)^{2}-\left(\theta-\xi_{1} e^{-a \theta+b}\right)\right) \frac{e^{-\frac{(\ln (\theta)-\mu)^{2}}{2 \sigma^{2}}}}{\sqrt{2 \pi} \sigma}\right)+ \\
+\int_{\frac{\ln (\theta)-\mu}{\sigma}}^{+\infty}\left(1+a \xi_{1} e^{-a \theta+b}+2 \xi_{1} \beta^{2}\right) \frac{1}{\sqrt{2 \pi}} e^{-\frac{1}{2} t^{2}} d t+ \\
+2 \xi_{1}\left(\int_{-\infty}^{\frac{\ln (\theta)-\mu}{\sigma}}\left(\alpha-x-\frac{1}{2 \xi_{1} \beta}\right) \frac{1}{\sqrt{2 \pi}} e^{-\frac{1}{2} t^{2}} d t\right)=0
\end{gathered}
$$

and

$$
\begin{gathered}
\left(\alpha-\left(\theta+\xi_{2} e^{-a \theta+b}\right)\right) \frac{e^{-\frac{(\ln (\theta)-\mu)^{2}}{2 \sigma^{2}}}}{\sqrt{2 \pi} \sigma}+\int_{\frac{\ln (\theta)-\mu}{\sigma}}^{+\infty}\left(2-a \xi_{2} e^{-a \theta+b}\right) \frac{1}{\sqrt{2 \pi}} e^{-\frac{1}{2} t^{2}} d t+ \\
+\int_{-\infty}^{\frac{\ln (\theta)-\mu}{\sigma}}\left(1-\frac{1}{\beta}\right)\left(1-2 \xi_{2}\left(\alpha-x-\frac{1}{2 \xi_{1} \beta}\right)\right) \frac{1}{\sqrt{2 \pi}} e^{-\frac{1}{2} t^{2}} d t=0
\end{gathered}
$$

where $\theta=\alpha-\frac{1}{2 \xi_{1} \beta}$. follows:

Solving the system of Equations (39) and (40), we obtain optimal solutions $\left(\alpha^{*}, \beta^{*}\right)$ as

$$
\begin{aligned}
g_{1}(\theta)= & \left(\left(\frac{1}{2 \xi_{1} \beta^{*}}-\xi_{1}\left(\frac{1}{2 \xi_{1} \beta^{*}}\right)^{2}+\xi_{1} e^{-a \theta^{*}+b}\right) \frac{e^{-\frac{\left(\ln \left(\theta^{*}\right)-\mu\right)^{2}}{2 \sigma^{2}}}}{\sqrt{2 \pi} \sigma}\right) \\
& +\left(1+a \xi_{1} e^{-a \theta^{*}+b}+2 \xi_{1} \beta^{* 2}\right)\left(1-\Phi\left(\frac{\ln \left(\theta^{*}\right)-\mu}{\sigma}\right)\right)+ \\
& +2 \xi_{1} \theta^{*} \Phi\left(\frac{\ln (\theta)-\mu}{\sigma}\right)-2 \xi_{1} e^{\mu+\frac{\sigma^{2}}{2}} \Phi\left(\frac{\ln (\theta)-\mu}{\sigma}-\sigma\right)=0
\end{aligned}
$$

$$
\begin{aligned}
g_{2}(\theta) & =\left(\frac{1}{2 \xi_{1} \beta^{*}}-\xi_{2} e^{-a \theta^{*}+b}\right) \frac{e^{-\frac{\left(\ln \left(\theta^{*}\right)-\mu\right)^{2}}{2 \sigma^{2}}}}{\sqrt{2 \pi} \sigma}+\left(2-a \xi_{2} e^{-a \theta^{*}+b}\right)\left(1-\Phi\left(\frac{\ln \left(\theta^{*}\right)-\mu}{\sigma}\right)\right) \\
& +\left(1-\frac{1}{\beta}\right)\left(1-2 \xi_{2} \theta^{*}\right) \Phi\left(\frac{\ln \left(\theta^{*}\right)-\mu}{\sigma}\right)+2 \xi_{2}\left(1-\frac{1}{\beta}\right) e^{\mu+\frac{1}{2} \sigma^{2}} \Phi\left(\frac{\ln \left(\theta^{*}\right)-\mu}{\sigma}-\sigma\right)=0
\end{aligned}
$$

where $\theta^{*}=\alpha^{*}-\frac{1}{2 \xi_{1} \beta^{*}}$ and $\Phi(x)$ is the cumulative distribution function of the standard normal distribution. Substituting these into Equation (37), combining with the Equation (38) and letting $t=\frac{\ln x-\mu}{\sigma}$ (so that we can work with the standard normal distribution), we obtain

$$
\begin{aligned}
E\left(I^{*}(s, x)\right)= & \int_{0}^{\theta} \frac{x+\frac{1}{2 \xi_{1} \beta}-(1-\beta) \alpha}{\beta} f(x) d x+\int_{\theta}^{+\infty} \theta f(x) d x= \\
& =\int_{-\infty}^{\frac{\ln (\theta)-\mu}{\sigma}} \frac{e^{t \sigma+\mu}+\frac{1}{2 \xi_{1} \beta}-(1-\beta) \alpha}{\beta} \frac{1}{\sqrt{2 \pi}} e^{-\frac{t^{2}}{2}} d t+\int_{\frac{\ln (\theta)-\mu}{\sigma}}^{+\infty} \theta e^{-\frac{t^{2}}{2}} d t= \\
& =\frac{\frac{1}{2 \xi_{1} \beta}-(1-\beta) \alpha}{\beta}\left(\Phi\left(\frac{\ln (\theta)-\mu}{\sigma}\right)\right)+ \\
& +e^{\mu+\frac{1}{2} \sigma^{2}} \frac{1}{\beta} \Phi\left(\frac{\ln (\theta)-\mu}{\sigma}-\sigma\right)+\theta\left(1-\Phi\left(\frac{\ln (\theta)-\mu}{\sigma}\right)\right) .
\end{aligned}
$$

We also obtain the expected inflated claim amount as:

$$
E\left(s^{*}(\beta)\right)=\int_{0}^{\theta}\left(x+\frac{1}{2 \xi_{1} \beta}\right) f(x) d x+\int_{\theta}^{+\infty} \theta f(x) d x .
$$


Let $t=\frac{\ln (x)-\mu}{\sigma}$. Then we have:

$$
\begin{gathered}
E\left(s^{*}(\beta)\right)=\int_{-\infty}^{\frac{\ln (\theta)-\mu}{\sigma}}\left(e^{t \sigma+\mu}+\frac{1}{2 \xi_{1} \beta}\right) \frac{1}{\sqrt{2 \pi}} e^{-\frac{t^{2}}{2}} d t+\int_{\frac{\ln (\theta)-\mu}{\sigma}}^{+\infty} \theta \frac{1}{\sqrt{2 \pi}} e^{-\frac{t^{2}}{2}} d t= \\
=\left(\frac{1}{2 \xi_{1} \beta}+\theta\right) \Phi\left(\frac{\ln (\theta)-\mu}{\sigma}\right)+e^{\mu+\frac{1}{2} \sigma^{2} \Phi\left(\frac{\ln (\theta)-\mu}{\sigma}-\sigma\right)}
\end{gathered}
$$

Equation (41) is the first order condition for the objective function to maximize the expected total wealth of the insured, while Equation (42) is the first order condition for the objective function to minimize the total expected loss of the insurer. Although it is difficult to explicitly prove that the first order conditions of Equations (41) and (42) are satisfied, we can find by numerical analysis that $\lim _{\theta(\alpha, \beta) \rightarrow \theta\left(w_{1}^{+}, v_{1}^{+}\right)} g_{1}(\theta(\alpha, \beta), \alpha, \beta)<0$ and $\lim _{\theta(\alpha, \beta) \rightarrow \theta\left(w_{1}^{+}, v_{1}^{+}\right)} g_{2}(\theta(\alpha, \beta), \alpha, \beta)>$ 0 while $\alpha \in\left(k_{1}, k_{2}\right)$ and $\lim _{\theta(\alpha, \beta) \rightarrow \theta\left(w_{2}^{-}, v_{2}^{-}\right)} g_{2}(\theta(\alpha, \beta), \alpha, \beta)<0$ in the feasible area of $\alpha \in$ $\left(w_{1}, w_{2}\right)$ and $\beta \in\left(v_{1}, v_{2}\right)$, both graphs of $g_{1}(\theta(\alpha, \beta), \alpha, \beta)$ and $g_{2}(\theta(\alpha, \beta), \alpha, \beta)$ cross the $x$ axis, and the graph of $g_{1}(\theta(\alpha, \beta), \alpha, \beta)$ intersects $\left.g_{2}(\theta(\alpha, \beta), \alpha, \beta)\right)$ and the $x$ axis at point A. Optimal solutions of $\left.\theta^{*}\left(\alpha^{*}, \beta^{*}\right), \alpha^{*}, \beta^{*}\right)$ exist, and are located at the point given by the condition

$$
g_{1}\left(\theta^{*}\left(\alpha^{*}, \beta^{*}\right), \alpha^{*}, \beta^{*}\right)=g_{2}\left(\theta^{*}\left(\alpha^{*}, \beta^{*}\right), \alpha^{*}, \beta^{*}\right)=0,
$$

with other given parameters applied: $a=1 / 1000, b=25, \xi_{1}=0.0003, \xi_{2}=0.005$.

In the following, we will explain the process of obtaining optimal solutions using the numerical method. In the feasible area of $\alpha \in\left(k_{1}, k_{2}\right)$ and $\beta \in\left(v_{1}, v_{2}\right)$ (where $0 \leq$ $\alpha<+\infty, 0<\beta<1)$, for each pair of $\alpha$ and $\beta$, calculate the values of $g_{1}(\theta(\alpha, \beta), \alpha, \beta)=$ $g_{2}(\theta(\alpha, \beta), \alpha, \beta)$ by using the constraint $E(s(\beta)) \leq E(I(s(\beta), \alpha, \beta)) \geq 0$ and the optimal solutions will be obtained at $g_{1}\left(\theta^{*}\left(\alpha^{*}, \beta^{*}\right), \alpha^{*}, \beta^{*}\right)=g_{2}\left(\theta^{*}\left(\alpha^{*}, \beta^{*}\right), \alpha^{*}, \beta^{*}\right)=0$.

Since the values of $g_{1}$ and $g_{2}$, when $\alpha \neq \alpha^{*}$ and $\beta \neq \beta^{*}$ but close to $\alpha^{*}$ and $\beta^{*}$, are of opposite signs, we calculate the value of $g=g_{1}+g_{2}$ for each pair of values of $\alpha$ and $\beta$, selected in the range of $\alpha \in\left(k_{1}, k_{2}\right)$ and $\beta \in\left(v_{1}, v_{2}\right)$ until $g \leq \varepsilon$ where $\varepsilon$ is a sufficiently small preset value. The optimal solutions of $\left(\theta^{*}, \alpha^{*}, \beta^{*}\right)$ will be those satisfying $g \leq \varepsilon$. In this way, we find the optimal solutions of $\left(\theta^{*}, \alpha^{*}, \beta^{*}\right)=(12100,13829,0.8694)$. Entering $\left(\theta^{*}, \alpha^{*}, \beta^{*}\right)$ into Equation (41), we obtain $E\left(I^{*}\left(s\left(\beta^{*}\right), \alpha^{*}, \beta^{*}\right)\right)=8494.6$. Figure 1 displays the patterns of change of the first order conditions of the two objective functions and optimal upper limit. Table 1 lists the optimal solutions when the parameters take different values.

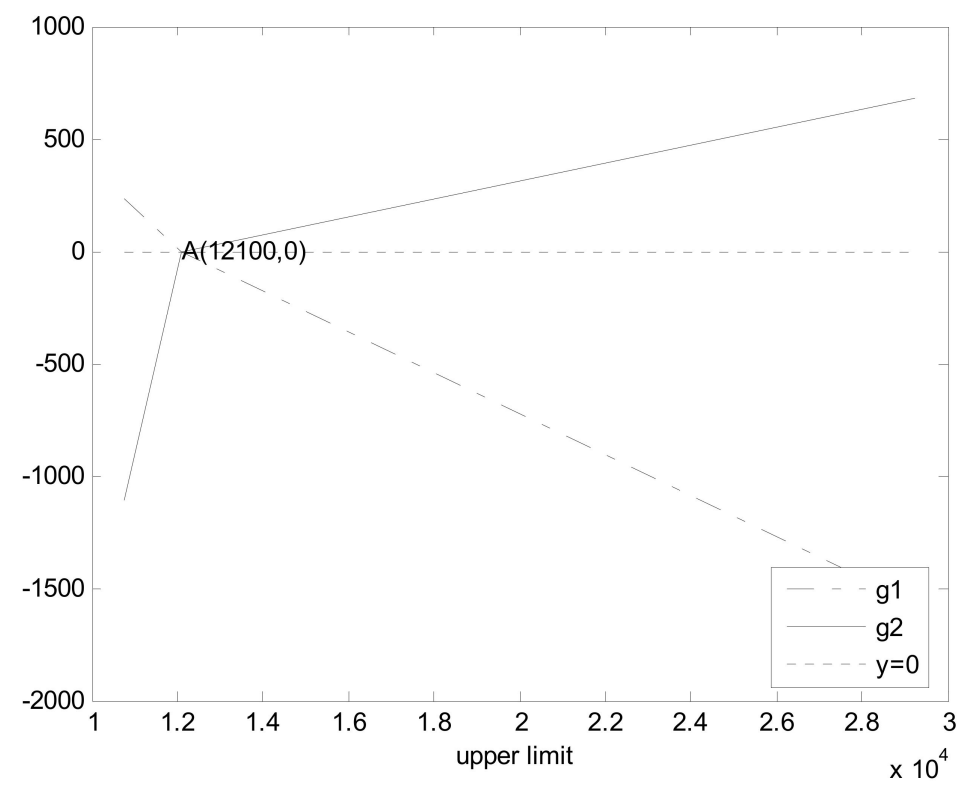

Figure 1. Patterns of changes in the first order conditions of the two objective functions and optimal upper limit. 
Table 1. Optimal solutions with different values of parameters.

\begin{tabular}{cccc}
\hline \multicolumn{4}{c}{$\xi_{1}=0.0006, \xi_{2}=0.009, a=1 / 1200, b=12$} \\
\hline$\alpha^{*}$ & $\beta^{*}$ & $\theta^{*}$ & $E^{*}(I)$ \\
\hline 13,000 & 0.94 & 12,113 & 8372.2 \\
\hline$\alpha^{*}$ & $\xi_{1}=0.0006, \xi_{2}=0.009, a=1 / 1000, b=25$ \\
\hline 14,000 & $\beta^{*}$ & $\theta^{*}$ & $E^{*}(I)$ \\
\hline \multicolumn{4}{c}{$\alpha_{1}=0.0003, \xi_{2}=0.005, a=1 / 1200, b=22$} \\
\hline 15,000 & $\beta^{*}$ & $\theta^{*}$ & 8818.8 \\
\hline & 0.36 & 10,370 & $E^{*}(I)$ \\
\hline
\end{tabular}

Note the following clarifications about Figure 1:

- The two lines represent two tangents of first order differentiation, and the point where these tangents cross the horizontal axis is the optimal solution.

- There is one maximization model and one dual minimization model. Letting the first order condition of both be equal to zero yields optimal solutions. In the maximization problem, its first order derivative in the left end point is greater than zero; however, its first order derivative in the right end point is less than zero. In the dual minimization problem, its first order derivative in the left end point is less than zero; however, in the right end point it is greater than zero. Each of two lines crosses the horizontal axis, and the cross point corresponds to the optimal value of the upper limit. In Figure 1, this is point $\mathrm{A}$.

\section{Conclusions}

In this paper, we discuss the optimization of claim settlement strategy for third party liability insurance, along with consideration of an upper limit. At the same time, we establish objective functions to maximize the claimant's net benefit from inflating the claim amount in order to maximize the expected total wealth of the insured. Then, we establish a dual objective function to maximize the expected loss of the insurer, so as to lessen a claimant's incentive to exaggerate losses, as well as to reduce the falsification costs and other indirect costs due to setting up the cap. Solving the two objective functions for the insured and the dual objective function for the insurer, we find the optimal indemnification schedule vs. optimal caps with different values of parameters.

We believe that our research work in this paper may provide effective claim settlement strategies which are favorable for all parties, including regulators, insurers, and the insured. Regulators may tend to favor damage award caps for reducing the insolvency of insurers and other indirect loss due to setting the cap. In order to protect consumer benefits, insurers are expected to reduce their payment of inflated claims, litigation and settlement costs, and other indirect cost by optimizing claim settlement strategies to motivate the insured to reduce their exaggeration of claimed losses; the insured will obtain fair compensation for their claimed loss, have the incentive to decline their falsification cost, and reduce the indirect loss resulting from setting up the cap.

Author Contributions: Conceptualization, H.M. and K.O.; methodology, H.M. and K.O.; software, H.M.; validation, H.M.; formal analysis, H.M. and K.O.; investigation, H.M. and K.O.; resources, H.M. and K.O.; data curation, H.M.; writing-original draft preparation, H.M.; writing-review and editing, H.M. and K.O.; visualization, H.M.; supervision, H.M. and K.O.; project administration, H.M. and K.O. funding acquisition, H.M. and K.O. All authors have read and agreed to the published version of the manuscript.

Funding: This research received no external funding. 
Institutional Review Board Statement: "Not applicable." for studies not involving humans or animals.

Informed Consent Statement: Not applicable.

Data Availability Statement: Not applicable.

Acknowledgments: We express our most sincere thanks for Liang Hong's most valuable and insightful suggestions in developing this paper.

Conflicts of Interest: The authors declare no conflict of interest.

\section{References}

1. Borch, K. Optimal insurance arrangement. Astin Bull. 1975, 8, 284-290. [CrossRef]

2. Cummins, J.D.; Mahul, O. The demand for insurance with upper limit on coverage. J. Risk Insur. 2004, 71, 253-264. [CrossRef]

3. Schlesinger, H. Mossin's theorem for upper-limit insurance policies. J. Risk Insur. 2006, 73, 297-301. [CrossRef]

4. Huang, R.; Tzeng, L. Optimal tax deduction for net losses under private insurance with upper limit. J. Risk Insur. 2007, 74, 883-893. [CrossRef]

5. Mao, H.; Ostaszewski, K.; Carson, J.; Wang, Y. Pricing of deposit insurance considering investment, deductibles, and policy limit. J. Insur. Issues 2013, 36, 149-174.

6. Crocker, K.J.; Morgan, J. Is Honesty the Best Policy? Curtailing Insurance Fraud Through Optimal Incentive Contracts. J. Political Econ. 1998, 106, 355-375. [CrossRef]

7. Crocker, K.J.; Tennyson, S. Insurance Fraud and Optimal Claims Settlement Strategies. J. Law Econ. 2002, 45, 469-507. [CrossRef]

8. Bambauer, J.; Roth, A. From damages caps to decarceration: Extending tort law safeguards to criminal sentencing. Boston Univ. Law Rev. 2021, 101, 1671-1721.

9. Klick, J.; MacDonald, J. Deterrence and liability for intentional torts. Int. Rev. Law Econ. 2020, 63, 105-126. [CrossRef]

10. Born, P. The Influence of Tort Reform on Auto Liability Losses and Premiums. J. Insur. Issues 2017, 40, 61-89.

11. Mert, O.; Selcuk-Kestel, A. Time dependent stop-loss reinsurance and exposure curves. J. Comput. Appl. Math. 2021, 389, 113348. [CrossRef]

12. Mao, H.; Ostaszewski, K.; Carson, J.; Wang, Y. Optimal cap on claim settlements based on social benefit maximization. J. Insur. Regul. 2016, 35, 1-23.

13. Mao, H.; Carson, J.; Ostaszewski, K.; Lou, Y.; Wang, Y. Determining the Insurer's Optimal Investment and Reinsurance Strategy Based on Stochastic Differential Game. J. Insur. Issues 2016, 39, 187-202.

14. Mao, H.; Carson, J.; Ostaszewski, K. Optimization of Dynamic Minimum Guarantee Return, Investment And Reinsurance Strategy By Balancing the Risks And Benefit of Both Insurers' and Consumers. J. Insur. Financ. 2016, 2, 81-105.

15. Mao, H.; Carson, J.; Ostaszewski, K. Optimal Insurance Pricing, Reinsurance, and Investment for a Jump Diffusion Risk Process Under a Competitive Market. J. Insur. Issues 2017, 40, 90-124.

16. Owen, G. Game Theory, 3rd ed.; Emerald Group Publishing Limited: Bingley, UK, 1995.

17. Abolmohammadi, M.; Seif, A.; Behzadi, M.H.; Moghadam, M.B. Economic statistical design of adaptive $\bar{X}$ control charts based on quality loss functions. Oper. Res. Int. J. 2021, 21, 1041-1080. [CrossRef] 